Pteridines

Vol. 1, 1989, pp. 65-70

\title{
Selective Cytotoxicity of Carboxypeptidase-activated Methotrexate $\alpha$-Peptides*
}

\author{
By Karin S. Vitols, Yolanda D. Montejano, Ulrike Kuefner** and F. M. Huennekens \\ Division of Biochemistry, Department of Basic and Clinical Research, Research Institute of Scripps Clinic, \\ La Jolla, CA 92037, U.S.A.
}

(Received September 1988)

\begin{abstract}
Summary
Methotrexate $\alpha$-peptides (derivatives in which an amino acid is linked covalently to the $\alpha$-carboxyl of the glutamate residue on the parent drug) can be hydrolyzed by specific carboxypeptidases to yield free Methotrexate (MTX) and the corresponding amino acid. Studies with L1210 cells in suspension culture have shown that the MTX peptides can serve as "pro-drugs": Because of their inability to be taken up by cells, they are relatively non-toxic. When co-administered with appropriate carboxypeptidases, however, they become equitoxic with MTX. In the present investigation, the potential of the MTX-ala/carboxypeptidase A combination for providing regional cytotoxicity was demonstrated in a model system involving L1210 cells propagated in semi-solid agarose. When cells and one of the components (MTX peptide or carboxypeptidase) were distributed uniformly throughout the agarose, and the other component was immobilized at the center, a discrete zone of cell kill radiated from the fixed component. These results suggest the possibility of developing a new mode of cancer chemotherapy involving circulating MTX peptides in conjunction with carboxypeptidases linked covalently to tumor-targeted monoclonal antibodies.
\end{abstract}

\section{Introduction}

Many agents employed in cancer chemotherapy are able to achieve total cell kill when tested under optimal conditions in laboratory culture systems. Similar results are difficult to obtain with tumors in vivo because the required drug levels produce unacceptable

* Manuscript BCR-5284 from the Research Institute of Scripps Clinic. This work was supported by an Outstanding Investigator Grant (CA-39836) from the National Institutes of Health and by a grant (CH-31) from the American Cancer Society. A preliminary account of this work was presented at the Seventh Winter Workshop on Pteridines, St. Cristoph. Austria (1).

** Recipient of a Postdoctoral Travel Fellowship from the Deutsche Forschungsgemeinschaft. Present address: Abteilung Pharmachemie, Boehringer Ingelheim KG, D-6507 Ingelheim. F.R. of Germany.

Enzymes used: carboxypeptidase A, peptidyl-L-amino-acid hydrolase (EC 3.4.17.1); carboxypeptidase B, peptidyl-L-lysine ( $L$ arginine) hydrolase (EC 3.4.17.2); carboxypeptidase $\mathrm{G}_{2}$ (EC 3.4.22.12); carboxypeptidase $\mathrm{N}$, peptidyl-L-arginine hydrolase (EC 3.4.17.3)

Abbreviations used: MTX, Methotrexate; MTX-ala, -asp, and arg, peptides in which the indicated amino acids are linked covalently to the $\alpha$-carboxyl of MTX; MTT, 3-(4,5-dimethylthiazol-2-yl)-2,5-diphenyl tetrazolium bromide; ID $_{50}$, concentration producing $50 \%$ cell kill.

Pteridines / Vol.1/1989/ No.

Copyright (C) 1989 Walter de Gruyter - Berlin - New York side-effects on normal tissues in the body. Use of suboptimal doses, while providing some benefit by decreasing the tumor burden, allows surviving cells to metastasize or drug-resistant cell populations to emerge. These problems might be circumvented if it were possible to expose tumor cells (but not normal cells) to lethal concentrations of the drugs. The strategy currently under investigation in this laboratory would employ derivatized drugs that are unable to enter either tumor or normal cells, but from which the parent drugs (readily taken up by cells) could be generated enzymatically in the vicinity of tumors. This approach is exemplified by studies on Methotrexate $x$-peptides (derivatives in which an amino acid is linked covalently to the $\alpha$-carboxyl of the drug) that are relatively non-toxic because of their inability to be transported into cells. These "pro-drugs" can be hydrolyzed, however, by carboxypeptidases to yield free Methotrexate (MTX). The present report describes a model system that demonstrates the feasibility of making this activation process regio-specific, i. e., localizing the cytotoxic effect of MTX within a population of cells. 

istic of reaction [2], viz., decreased absorbance at 320 $\mathrm{nm}$. This procedure was used, for example, to determine the $\mathrm{K}_{\mathrm{m}}(0.5 \mathrm{mM})$ for hydrolysis of MTX-ala with carboxypeptidase $\mathrm{A}$.

Cytotoxicity of MTX peptides toward L1210 cell in suspension culture

Using the MTT assay (see Material and Methods), cells were grown in the presence of varying concentrations of the MTX peptides and appropriate carboxypeptidases. Results were plotted as the percent of surviving cells vs. drug concentration; typical sigmoidal cell-kill curves were obtained (see Fig. 8 in ref. (2)). ID $_{50}$ values from a representative experiment in which carboxypeptidase $\mathrm{A}$ was used to activate MTX-ala are summarized in Table 1. MTX-ala was ca. 100-fold less toxic than MTX. Inclusion of carboxypeptidase $\mathrm{A}$ in the medium, however, considerably improved the toxicity of the MTX peptide. At the concentrations used, carboxypeptidase A itself was non-toxic (data not shown). Similar results, described elsewhere $(2,3)$, were obtained with MTXasp/carboxypeptidase A, MTX-ala/carboxypeptidase B, MTX-arg/carboxypeptidase B and MTX-arg/carboxypeptidase $\mathrm{N}$ combinations.

Table 1. Carboxypeptidase A-mediated cytotoxicity of MTXala

\begin{tabular}{ll}
\hline Conditions & $\mathrm{ID}_{50}$ \\
\hline & $\mu \mathrm{M}$ \\
MTX & 0.024 \\
MTX-ala & 2.0 \\
$\begin{array}{l}\text { MTX-ala }+ \text { carboxypeptidase A } \\
(7.8 \mathrm{mU} / 1.5 \mathrm{~mL})\end{array}$ & 0.20 \\
$\begin{array}{l}\text { MTX-ala }+ \text { carboxypeptidase A } \\
(15.7 \mathrm{mU} / 1.5 \mathrm{~mL})\end{array}$ & 0.085 \\
\hline
\end{tabular}

L1210 cells were grown for 3 days in the presence of various concentrations of MTX or MTX-ala and. in the latter instance, with the indicated concentrations of carboxypeptidase $A$. One unit of carboxypeptidase A will hydrolyze $1 \mu \mathrm{mol}$ min of hippurylphenylalanine at $\mathrm{pH} 7.5$ and 25 . For other details. see Material and Methods.

\section{Regional cytotoxicity toward immobilized cells}

To ascertain whether MTX-ala in conjunction with carboxypeptidase A could achieve regio-specific toxicity, a model system was employed in which the cells were immobilized in semi-solid agarose and exposed to various spatial configurations of the enzyme and MTX peptide. The first experiment was designed to examine the general parameters of cell growth under these conditions, including morphologic characteris-

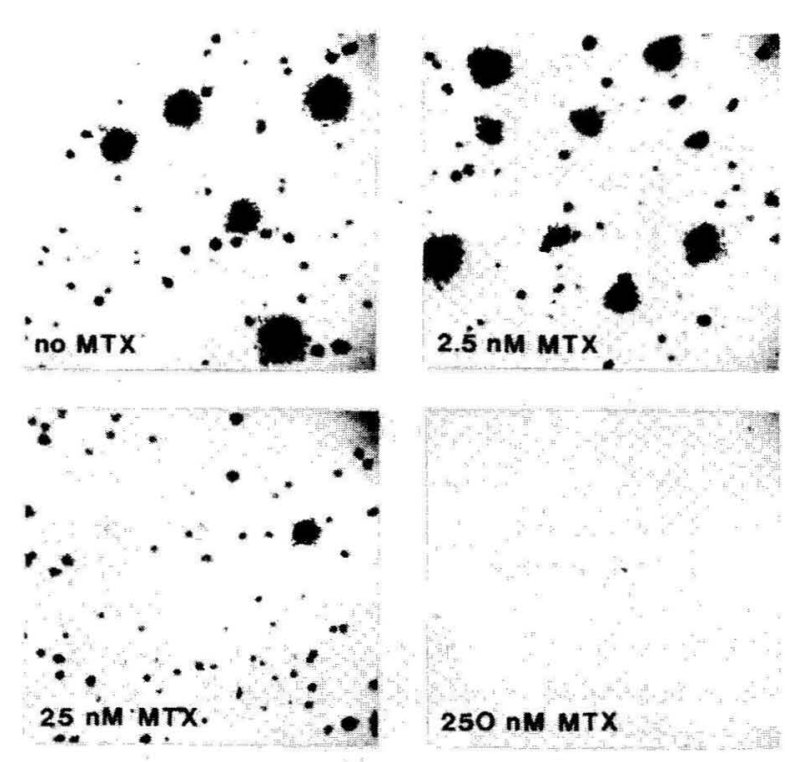

Figure 3. Effect of MTX on L1210 cell colony growth on semisolid agarose. Cells were cultured in the 2-layer system as described in Material and Methods with the indicated concentrations of MTX included in the agarose. Dishes were photographed using a Zeiss phase-contrast fluorescence microscope (bright field; front lens removed; Polaroid 667 film; 1/60th s exposure).

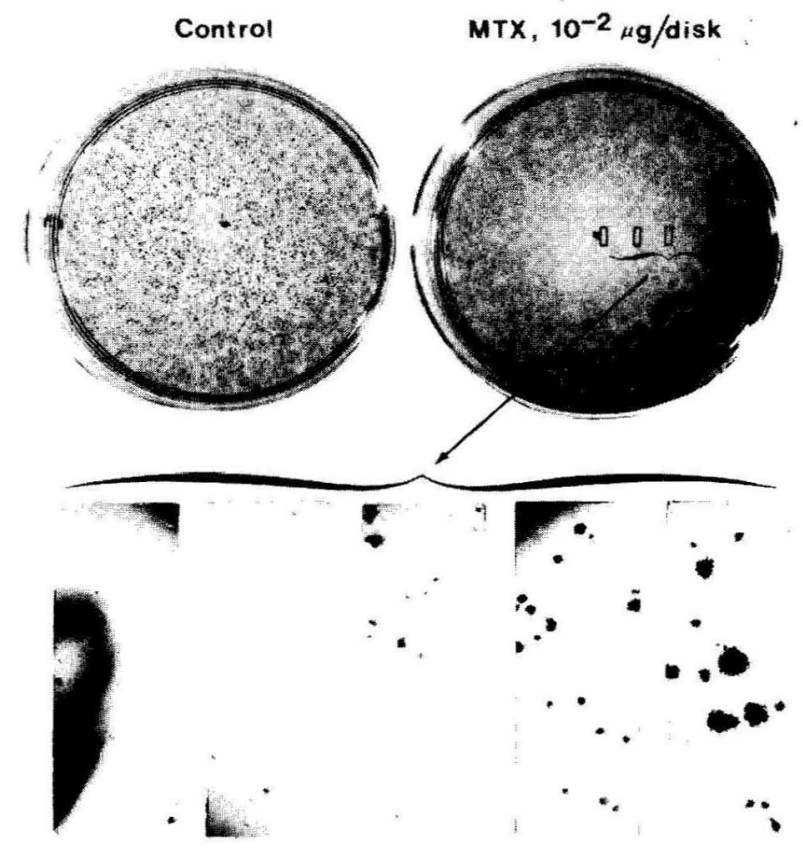

Figure 4. Regio-specific toxicity of MTX toward L1210 cell growth. Conditions as in Figure 3, except that MTX $\left(10^{-2} \mu \mathrm{g}\right.$ in $2 \mu \mathrm{L}$ ) was added to a filter paper disk (Whatman No. $1 ; 0.6$ $\mathrm{cm}$ diameter) which was floated at the center of the top layer (prior to photography, the disk was removed and the center marked with a pen on underside of the dish). Control: disk with $2 \mu \mathrm{l}$ of phosphate-buffered saline. Top portion of figure: photographs of dishes. Lower portion of figure: photographs through the microscope at the indicated locations on the MTX dish. 
tics of the colonies and the effect of MTX. L1210 cells were seeded at high density $\left(10^{5}\right.$ cells per Petri dish) in semi-solid agarose containing RPMI 1640, fetal bovine serum, antibiotics, and various concentrations of MTX. Results are shown in Figure 3. In the absence of drug, large, actively-growing colonies, which stained dark blue upon addition of the MTT, were formed. MTX (at $2.5 \mathrm{nM}$ ) had little effect on cell proliferation. Fewer colonies appeared, however, when the concentration of the drug was raised 10fold, and growth was completely suppressed at 250 nM MTX.

In the following experiment, MTX was placed on a filter paper disk located at the center fo the dish (Fig. 4). Univorm growth was observed in the control (no MTX). In the presence of the drug, conversely, a clear zone indicative of cell kill radiated from the disk. The lower panels in this Figure are photographs taken through the microscope at the indicated locations between the disk and the edge of the dish. Large colonies appeared only at the outer edge. This result demonstrates that the drug is released from the disk and diffuses slowly through the semi-solid medium.

Based upon these results, a series of experiments was undertaken to investigate the regio-specific activation of MTX-ala. First, MTX-ala was placed on the centrally located filter paper disk, and carboxypeptidase A was distributed uniformly through the agarose (Fig. 5 ). Growth was uniform in the control (no carboxypeptidase-A), verifying that MTX-ala was non-toxic. In the carboxypeptidase A-containing dish, cell kill occurred in the zone where the mobile MTX-ala encountered the enzyme. Colonies near the edge of the

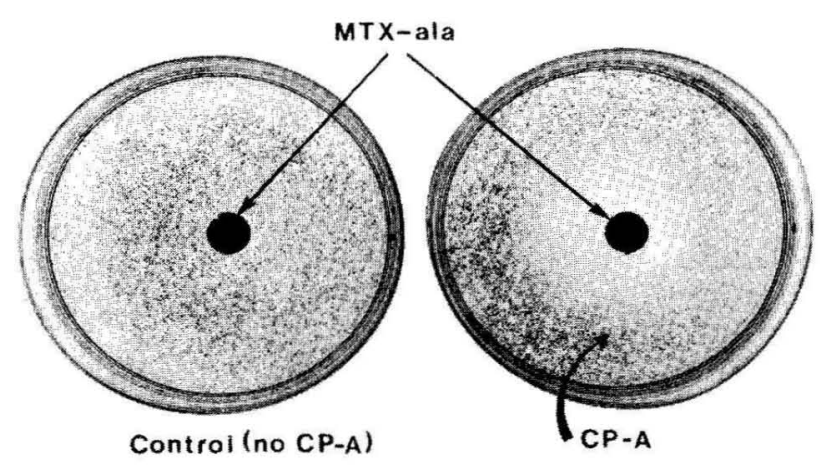

Figure 5. Regio-specific toxicity of MTX-ala/carboxypeptidase A. Conditions as in Figure 4, except that MTX was replaced on the disks by MTX-ala ( $0.1 \mu \mathrm{g}$ in $2 \mu \mathrm{L})$ and carboxypeptidase A $(0.3 \mathrm{U} / \mathrm{mL})$ was present in the agarose. Carboxypeptidase $\mathrm{A}$ solutions (commercial preparation diluted in sterile saline, rather than filter-sterilized) were added to the warm agarose immediately before dishes were poured, thereby minimizing exposure of the enzyme to elevated temperatures. Disks not removed for photography.

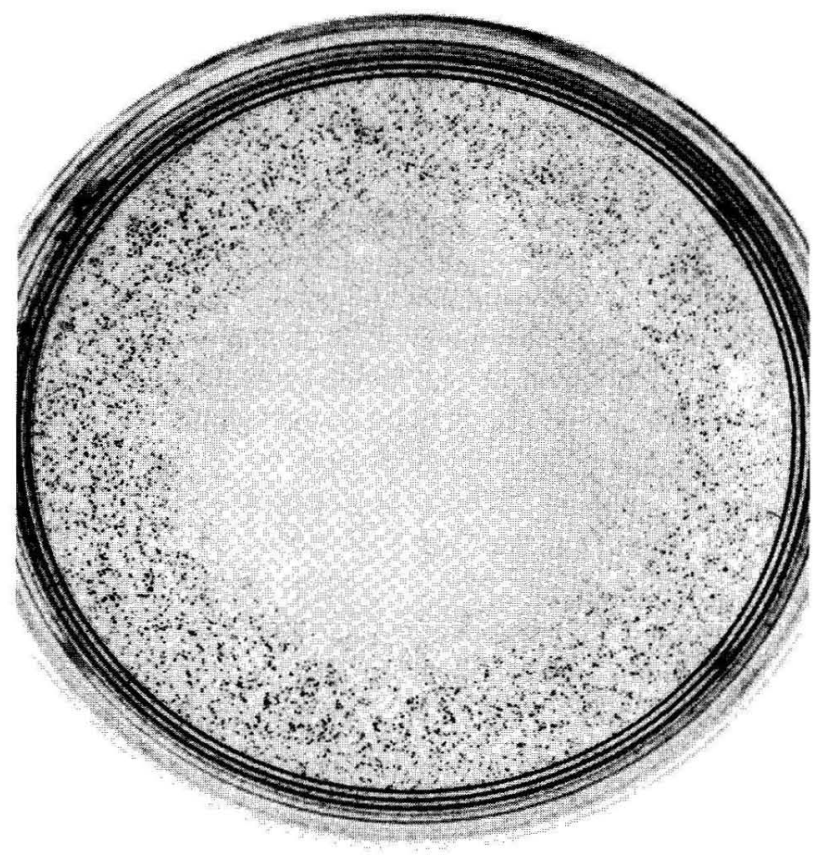

Figure 6. Regio-specific toxicity of MTX-ala in the presence of carboxypeptidase A immobilized on beads located in the center of the agarose dish. Conditions as in Figure 5 except that MTX-ala $\left(5 \times 10^{-7} \mathrm{M}\right)$ was present in the medium, and disks were not used. Carboxypeptidase A beads were washed with sterile saline and air-dried on a Whatman \# 1 filter paper under a laminar flow hood. A well was made by gently removing a plug from both layers of the agarose with the use of a sterile $10-\mathrm{mL}$ pipet. The beads, on a sterilized micro-spatula, were placed carefully in the well.

dish were unaffected. This experiment provided assurance that carboxypeptidase A retained catalytic activity even when relatively restricted in a semi-solid medium and that, at the amount employed, it was non-toxic to cells. In the reverse experiment (carboxypeptidase A on the disk and MTX-ala present in the agarose) regional cell kill was also observed (data not shown). It is not yet clear whether this latter result is due to diffusion of the enzyme from the disk and/or diffusion of MTX-ala toward the disk, followed by counter-diffusion of its product (free MTX).

Complete immobilization of the activating enzyme was achieved by the use of beads containing covalently-linked carboxypeptidase A. These beads were placed in a small well in the bottom layer at the center of the dish, and MTX-ala was present throughout the agarose (Fig. 6). The clear zone in the center verified that diffusion of MTX-ala and the liberated MTX was sufficiently rapid (relative to the division time of these cells) to achieve appreciable cell kill. As an extension of this experiment, the carboxypeptidase Acontaining beads were distributed randomly in the agarose (Fig. 7). Dense growth appeared in each of 


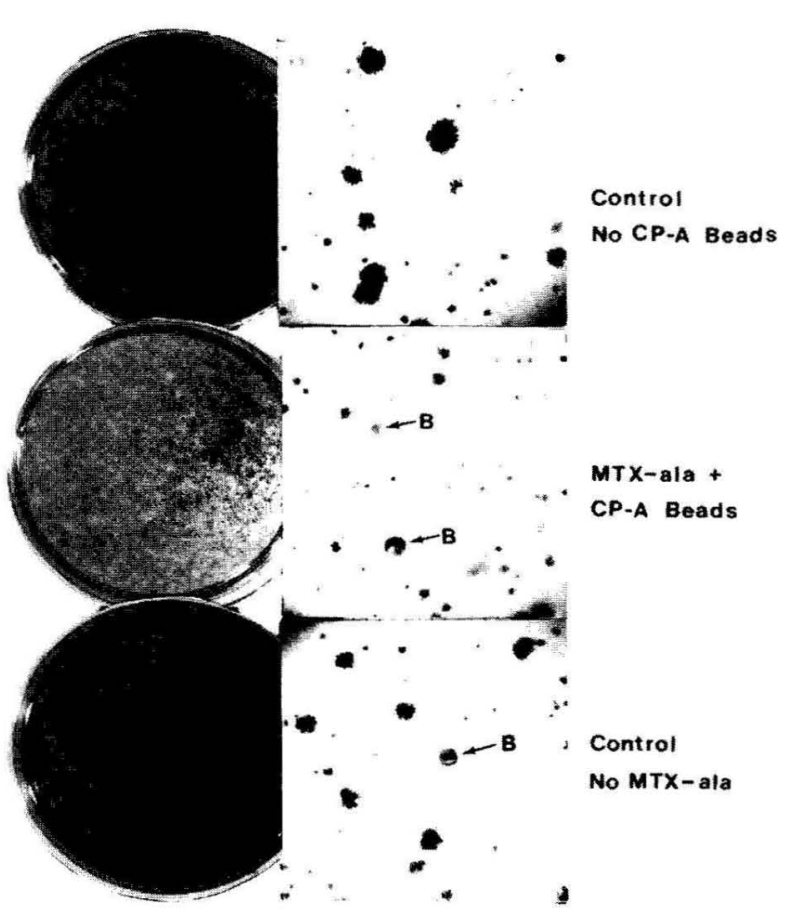

Figure 7. Regio-specific toxicity of MTX-ala in the presence of carboxypeptidase A immobilized on beads distributed randomly on the surface of the agarose. Conditions as in Figure 6 , except that the carboxypeptidase A-containing washed beads were suspended in sterile saline ( $1 \mu \mathrm{L}$ packed beads $/ \mathrm{mL}$ saline), and a $200 \mu \mathrm{L}$ aliguot (ca. $10^{3}$ units) was gently layered on the top surface of the semi-solid agarose.

the controls (no carboxypeptidase A or no MTX-ala), while appreciable cell kill was observed in the complete system.

\section{Future directions}

Previous results $(2,3)$ and those reported in this investigation have demonstrated that MTX- $x$-peptides are relatively non-toxic but that. in the presence of carboxypeptidases, they are converted to free MTX (a potent chemotherapeutic agent) in a regio-specific manner. Solid tumors with a localized mass of cells would appear to be amenable to this type of treatment, particularly if the activating enzyme could be delivered to the target via covalent linkage to a tumorspecific monoclonal antibody (Fig. 8). Unlike conventional monoclonal drug therapy in which each antibody molecule can deliver only a few drug molecules, a single carboxypeptidase molecule could generate a continuous supply of active drug in the immediate vicinity of the tumor cells. This method would also not require endocytic uptake and lysosomal hydrolysis of the antibody conjugate.

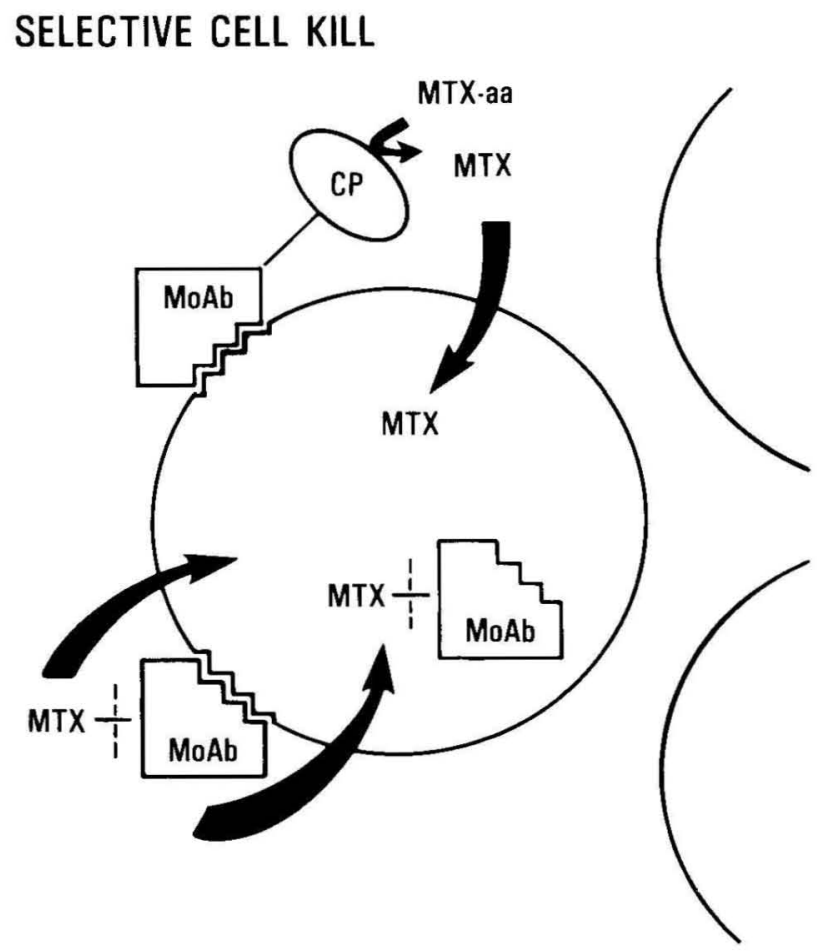

Figure 8. Schematic representation of selective cell kill using carboxypeptidase linked to tumor-specific monoclonal antibodies $(\mathrm{MoAb})$.

\section{Acknowledgements}

The authors are indebted to Dr. Laura Pope for advice concerning the semi-solid agarose assay and to Ms. Susan Burke for typing the manuscript.

\section{References}

1. Vitols, K. S., Montejano, Y. D., Lohrmann, U., Kuefner, U. \& Huennekens, F. M. (1988) Biol. Chem. Hoppe-Seyler 369,545 .

2. Kuefner, U., Lohrmann, U., Montejano, Y., Vitols, K. S. \& Huennekens, F. M. (1987) Adv. Enzyme Regul. 27, 3-13.

3. Kuefner, U., Lohrmann, U., Montejano, Y. D., Vitols, K. S. \& Huennekens, F. M. (1987) Submitted to Biochemistry.

4. Mosmann, T. (1983) J. Immunol. Methods 65, 55-63.

5. Finlay. G. J., Wilson, W. R. \& Baguley, B. C. (1986) Eur. J. Cancer Clin. Oncol, 22, 655-662.

6. Sirotnak. F. M., Chello, P. L., Piper, J. R., Montgomery, J. A. \& Degraw, J. I. (1979) in: Chemistry and Biology of Pteridines (Kisliuk, R. L. \& Brown, G. M., eds.) pp. 597 608. Elsevier North Holland, New York.

7. Rosowsky, A., Forsch, R., Uren, J. \& Wick, M. (1981) J. Med. Chem. 24, 1450-1455.

8. Piper. J. R.. Montgomery, J. A., Sirotnak, F. M. \& Chello, P. L. (1982) J. Med. Chem. 25, 182-187.

9. Sherwood. R. F.. Melton, R. G., Alwan, S. M. \& Hughes, P. (1985) Eur. J. Biochem. 148, 447-453. 